\title{
Sensitivity and Specificity of Sheard and Percival's Criteria for the Diagnosis of Young People with Near-heterophoria
}

\author{
Saif Hassan Alrasheed ${ }^{1}$, Amel Mohamed Yousif ${ }^{2}$, Majid A. Moafa ${ }^{1}$ D , Abd Elaziz Mohamed Elmadina ${ }^{1 *}$, Mohammad A. Alobaid ${ }^{1}$ D \\ ${ }^{1}$ Department of Optometry, College of Applied Medical Sciences, Qassim University, Buraydah, Saudi Arabia; ${ }^{2}$ Department of \\ Binocular Vision, Faculty of Optometry and Visual Sciences, Al-Neelain University, Khartoum, Sudan
}

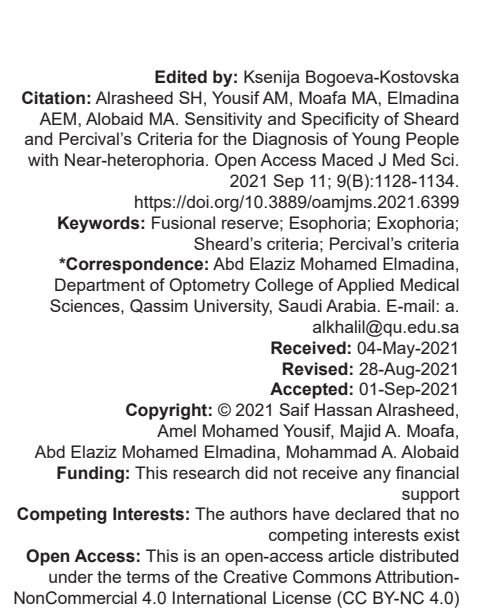

\section{Introduction}

The tendency of the eyes to move away from bi-foveal fixation (phoria) is controlled by fusional vergence; Positive Fusional Vergence (PFV) controlling exophoric deviation; and Negative Fusional Vergence (NFV) controlling esophoric deviation [1], [2], [3]. The fusional vergence is the amount of convergence and divergence that could be induced before fusion is lost and blurred or double vision happens [2], [3], [4]. Fusion types, sensory fusion is the ability of individuals to perceive an image formed on each eye at the same time and motor fusion is the ability of both eyes to maintain sensory fusion through a range of vergence movement, both types of fusion are innervated by third cranial nerve [5], [6], [7].

Fusional vergence is normally measured with rotary or variable prism devices and most commonly with a prism bar [1], [2]. Several methods have been recommended for the assessment of fusional vergence and these could be generally classified into intersubject and intrasubject [1]. Intersubject methods are based on the comparison of the results of fusional vergence of subjects with normative values [1], [8]. However, intrasubject techniques compare a person's fusional reserves with some other measure of that individual's binocular function [1], [4]. The first intrasubject method was initiated by Percival who postulated that fusional vergence should be balanced within the limits that one should not be less than half the other to overcome phoria symptoms [5], [9]. Percival's criterion seems to be suitable for diagnosis near heterophoria only; this method does not take considerations of the heterophoria size [1], [10].

The second intrasubject method was introduced by Sheard this technique related the heterophoria to its opposing fusional vergence blur point. It is stated that the opposing fusional reserve to the blur point should be at least twice the size of the phoria [1], [11], [12]. Sheard assumed that symptoms from heterophoria (latent strabismus) can be avoided if the fusional reserves in the opposite direction are at least twice the size of the phoria [1], [4], [8]. For instance, if a patient has $6 \Delta$ of exophoria deviation at near fixation then during PFV measurement (with base-out $\Delta$ ), he or she should not report blurring or diplopia until the prism exceeds $12 \Delta$ in compensated condition [1], [9]. 
Sheard's criterion can be used to prescribe prisms as well as to diagnose decompensated heterophoria. The concept is that a prism is required that will just cause the patient to pass Sheard's criterion or to decrease the heterophoria to less than half the opposing fusional vergence. Thus, if a patient has an exophoria of $6 \Delta$ and PFV of $8 \Delta$ then they would need a prism of $2 \Delta$ base-in. This prism should decrease the exophoria to $4 \Delta$ and increase the PFV to $10 \Delta$, therefore, Sheard's criterion would just be met [13], [14], [15].

Several authors [9], [14], [15] reported there are numerous problems when dealing with Sheard's criterion. The size of heterophoria differs depending on which dissociation test is used and fusional vergence is also highly dependent on the test conditions. However; investigational evidence suggested that Sheard's criterion has some value, mainly for exophoria at distance fixation and Percival's criterion has some value, particularly for esophoria at near fixation [1], [9], [16]. Sheedy and Saladin reported that Sheard's criterion was the best predictor of symptoms and Percival's criterion was useful for esophoric patients [17], [18], [19]. Measuring fusional vergence (PFV and NFV) has important diagnostic value to provide information about the ability to maintain binocular single vision [20], [21]. Heterophoria (latent strabismus) is controlled by fusional vergence; positive fusional reserves controlling exophoric deviation and negative fusional reserves controlling esophoric deviation [20], [22], [23]. Therefore, the current study aimed to determine the sensitivity and specificity of Sheard's and Percival's criteria for diagnosis young subjects with heterophoria.

\section{Materials and Methods}

\section{Study design}

This was descriptive of a cross-sectional hospital-based study of 230 heterophoric subjects were performed at Al-Neelain Eye Hospital Khartoum, from February to October 2019, all subjects underwent an eye examination due to ocular discomfort. The participants voluntarily visited a university eye hospital for primary eye care services.

\section{Inclusion and exclusion criteria}

Participants their age from 15 to 30 years old had not previously ocular history of any types of vision therapy and had not amblyopia or strabismus. Participants also were required to be near-emmetropes with no ocular or systemic clinical findings or use of medications that may cause ocular symptoms. Emmetropia was defined as a refractive error between
-0.50 dioptre (D) and $+0.75 \mathrm{D}$ spherical and cylinder $<0.25 \mathrm{D}$. The study excluded subjects with ocular diseases such as inflammation of the external eye, cataract, glaucoma, and retinal disease, and those with a history of a previous surgery.

\section{Ethical consideration}

Ethical approval was obtained from Al-Neelain University and the study was performed according to the Declaration of Helsinki guidelines. Informed consent was obtained from all subjects, however, the children their age $<18$ years old their permission was got from their parents/guardians to participate in this study. Participation in the study was voluntary and subjects were informed that they can withdraw from the study at any time without giving any reason. All forms and data sheets were shredded as soon as it is entered into the database system for analysis.

\section{Data collection procedures}

At the first, all participants underwent a case assessment to obtain information about the ocular history and complaints, followed by measurement of visual acuity at distance using Snellen Tumbling E-chart. Objective refraction was measured using a retinoscope (Neitz RX, Japan). A cover test was performed at $33 \mathrm{~cm}$ to reveal any heterophoria and to measure the size of deviation using Maddox Wing. The subjects underwent motility tests to assess the integrity of the eye muscles. The PFV and NFV were measured using a prism bar at $33 \mathrm{~cm}$. The prim bar was moved downwards at the speed of about one step per two seconds until the fixation object became a blur when the "blur point" was reached, the first prism value at which the subject was unable to see the target clear was registered as the blur point. Thereafter, the prism power slowly increases until the fixation object became double when the "breakpoint" was reached, the first prism value at which the subject was unable to fuse the target was registered as the breaking point. By moving the prism bar in the opposite direction, a recovery point was registered when the subject was able to fuse the object or see one object. All measurements were taken in a general clinical room by the same examiner, who performed all tests within approximately $30 \mathrm{~min}$, using the same methodology. Thereafter, the study applied Sheard's and Percival's criteria for diagnosing near heterophoria.

\section{Diagnosis near heterophoria using Sheard and Percival criteria}

Sheard criterion suggested that PFV and NFV to blur point should be at least twice the amount of exophoria and esophoria respectively to be compensated. In this study, we considered that if opposite fusional vergence (PFV for exophoria and 
NFV for esophoria) is double the size of phorias and the subject was asymptomatic or the fusional vergence less than double the amount of phoria and subject was symptomatic the Sheard criterion was successful in diagnosis. Otherwise, the Sheard criterion was failed in diagnosis near heterophoria.

Percival postulate that PFV for exophoria should not be less than half the NFV, while NFV for esophoria should not be less than half the PFV to overcome phoria symptoms. In the present study, we considered that if the PFV for the exophoric subject more than half NFV and/or NFV for esophoric subjects more than half of PFV and subject asymptomatic or the PFV for exophoric subject less than half NFV and/or NFV for esophoric subject less than half of PFV and subject was symptomatic then Percival Sheard criterion was successful in diagnosis. Otherwise, the criterion will have failed in diagnosis near heterophoria.

\section{Determining sensitivity, specificity, and predictive values}

In this study, to evaluate the accuracy of Sheard and Percival criteria, we use a sample of subjects who have resulted from both the criteria. The authors assume that Sheard's criteria are 100\% accurate for determining decompensated heterophoria at near (Phoria+) or absence (phoria-). For a test that yields binary test results (i.e., test positive $[A+B]$ or negative $[\mathrm{C}+\mathrm{D}])$, results were summarized in a $2 \times 2$ Table (Table 1) and calculated in (Tables 2 and 3 ).

Table 1: A $2 \times 2$ Table for comparing results from Sheard and Percival criteria

\begin{tabular}{llll}
\hline Screening test results & \multicolumn{2}{l}{ Decompensated heterophoria } & Total $(n)$ \\
\cline { 2 - 3 } & Phoria $+(n)$ & Phoria- $(n)$ & \\
\hline Positive & A & B & $(A+B)$ \\
Negative & C & D & $(C+D)$ \\
Total & $(A+C)+$ & $(B+D)-$ & $(A+B+C+D)$ \\
\hline
\end{tabular}

A True positive (near decompensated heterophoria correctly diagnosed by the criteria); B False-positive (healthy people wrongly diagnosed as near decompensated heterophoria); C False-negative (near decompensated phoria wrongly diagnosed as healthy); D True negative (healthy people correctly decompensated phoria diagnosed as healthy).
d

Two basic measures of test accuracy, sensitivity, and specificity, were estimated from the values in Table 1. Sensitivity is the test's ability to detect the disease when the disease is present

Table 2: Sensitivity and specificity of Sheard's criterion among exophoric and esophoric subjects

\begin{tabular}{llll}
\hline Exophoric & Frequency & Sensitivity & Positive predictive value \\
\hline True positive & 116 & $=87.2 \%$ & $=65.5 \%$ \\
False positive & 61 & Sepcificity & Negative predictive value \\
False-negative & 17 & $=8.0 \%$ & $=26 \%$ \\
True negative & 6 & Sensitivity & Positive predictive value \\
\hline Esophoric & Frequency & & \\
\hline True positive & 14 & $=77.8 \%$ & $=56 \%$ \\
False positive & 11 & Sepcificity & Negative predictive value \\
False-negative & 4 & & \\
True negative & 1 & $=9.0 \%$ & $=20 \%$ \\
Total & 230 & &
\end{tabular}

$$
=\frac{\text { true positive }}{\text { true positive }+ \text { false negative }}=\frac{A}{A+C}
$$

Specificity is the test's ability to exclude the disease when the disease is absent

$$
=\frac{\text { true negative }}{\text { true negative }+ \text { false positive }}=\frac{D}{D+B}
$$

The positive predictive value or precision rate is defined as a proportion of people with a positive test result $(24,25)$ in this study subjects with decompensated heterophoria $(A+B)$. It is calculated by the formula:

$$
=\frac{\text { true positive }}{\text { true positive }+ \text { false positive }}=\frac{A}{A+B}
$$

Table 3: Sensitivity and specificity of percival's criterion amongst exophoric and esophoric subjects

\begin{tabular}{llll}
\hline Exophoric & Frequency & Sensitivity & Positive predictive value \\
\hline True positive & 91 & $=67.4 \%$ & $=61.9 \%$ \\
False positive & 56 & Sepcificity & Negative predictive value \\
False-negative & 44 & $=13.8 \%$ & $=17 \%$ \\
True negative & 9 & Sensitivity & Positive predictive value \\
& & $=84.2 \%$ & $=61.5 \%$ \\
\hline Esophoric & Frequency & Sepcificity & Negative predictive value \\
\hline True positive & 16 & & \\
False positive & 10 & $=9.1 \%$ & $=25 \%$ \\
False-negative & 3 & & \\
True negative & 1 & &
\end{tabular}

The negative predictive value is defined as a proportion of people with a negative test result $(20,21)$ in this study subjects with compensated heterophoria $(\mathrm{C}+\mathrm{D})$. The formula for this measure is:

$$
=\frac{\text { true negative }}{\text { true negative }+ \text { false negative }}=\frac{D}{D+C}
$$

All the values were calculated in result section (Tables 2 and 3 ).

\section{Data analysis}

Statistical analysis was performed with SPSS for Windows Version 25.0 (SPPS Inc., Chicago, IL, USA). Means and standard deviations for phorias, PFV blur, break, and recovery points at $33 \mathrm{~cm}$ are reported for the 230 subjects. A one-way ANOVA test was used to compare means variables. A value of $p<0.05$ was considered statistically significant.

\section{Results}

\section{Socio-demographic characteristics of participants}

The demographic characteristics of the participants were as the following: The sample consisted 
of 230 participants of the $123(53.5 \%)$ females and $107(46.5 \%)$ males. The mean age of participants was $19.34 \pm 3.325$ years. The majority of subjects fell within the (15-20) year age group $154(67 \%)$ followed by 65 (21-25) year age group $11(28.3 \%)$, whereas the least number of participants were in the age group of 26-30 years $(4.8 \%)$.

\section{participants \\ Ocular deviation and complaints among}

The most common heterophoria was exophoria $200(87 \%)$ for near, the most ocular complaints were asthenopia 122 (53\%). The ocular complaints among esophoric and exophoric subjects were statically not significant $p=0.735$ as illustrated in Table 4 .

Table 4: Ocular complaints among exophoric and esophoric subjects

\begin{tabular}{lllll}
\hline Complaints & \multicolumn{2}{l}{ Direction of heterophoria } & Total $\mathrm{n}(\%)$ & $\mathrm{p}$-value \\
\cline { 2 - 4 } & Exophoria $\mathrm{n}(\%)$ & Esophoria $\mathrm{n}(\%)$ & & \\
\hline Visual perceptual distortion & $23(10)$ & $3(1.3)$ & $26(11.30)$ & \\
Binocular factors & $7(3.0)$ & $0(0.0)$ & $7(3.0)$ & 0.735 \\
Asthenopic symptoms' & $106(46)$ & $16(7.0)$ & $122(53)$ & \\
No symptoms & $64(27.8)$ & $11(4.8)$ & $75(32.6)$ & \\
Total & $200(87)$ & $30(13)$ & $230(100)$ & \\
\hline
\end{tabular}

\section{Sheard's criterion among exophoric and esophoric subjects}

The sheared postulate that PFV to blur point should be at least double the size of exophoria to be compensated. When this standard was used to diagnose exophoric subjects, the criterion was met in $177(88.5 \%)$ and unmet in $23(11.5 \%)$. The mean difference size of exophoria among subjects who met and unmet Sheard's criterion was not significant $p=0.608$. Regarding (opposing fusional vergence) PFV, the mean difference to blur and recovery points was not significant. However, the mean difference for PFV to breakpoint was statistically significant $p=0.021$, as shown in Table 5.

Table 5: Sheared and Percival criteria among exophoric subjects

\begin{tabular}{|c|c|c|c|}
\hline Sheared criterion & $\begin{array}{l}\text { Successful in diagnosis } \\
177(88.5 \%)\end{array}$ & $\begin{array}{l}\text { Failed in diagnosis } 23 \\
(11.5 \%)\end{array}$ & p-value \\
\hline Variable & [Mean \pm SD] & [Mean \pm SD] & \\
\hline Size of exophoria & {$[5.2 \pm 4.8 \Delta$ base-in] } & {$[5.7 \pm 2.2 \Delta$ base-in $]$} & 0.623 \\
\hline PFV (blur point) & {$[22.9 \pm 10.8 \Delta$ base-out $]$} & {$[24.0 \pm 8.9 \Delta$ base-out $]$} & 0.608 \\
\hline PFV (breakpoint) & {$[19.2 \pm 6.6 \Delta$ base-out $]$} & {$[15.9 \pm 5.7 \Delta$ base-out $]$} & 0.021 \\
\hline PFV (recovery point) & {$[25.3 \pm 10.3 \Delta$ base-out $]$} & {$[27.5 \pm 9.3 \Delta$ base-out $]$} & 0.44 \\
\hline Percival criterion & $\begin{array}{l}\text { Successful in diagnosis } \\
147(73.5 \%)\end{array}$ & $\begin{array}{l}\text { Failed in diagnosis } \\
53(26.5 \%)\end{array}$ & $p$-value \\
\hline Variable & {$[$ Mean \pm SD] } & {$[$ Mean \pm SD] } & \\
\hline Size of exophoria & {$[4.4 \pm 4.4 \Delta$ base-in $]$} & {$[7.7 \pm 5.2 \Delta$ base-in] } & 0 \\
\hline PFV (blur point) & {$[24.5 \pm 10.2 \Delta$ base-out $]$} & {$[18.6 \pm 10.5 \Delta$ base-out] } & 0 \\
\hline PFV(break point) & {$[26.9 \pm 9.7 \Delta$ base-out $]$} & {$[21.8 \pm 10.4 \Delta$ base-out $]$} & 0.002 \\
\hline PFV (recovery point) & {$[21.6 \pm 10.0 \Delta$ base-out $]$} & {$[16.5 \pm 10.1 \Delta$ base-out $]$} & 0.001 \\
\hline
\end{tabular}

Sheard assumed that symptoms from esophoria can be avoided if NFV is at least twice the size of the esophoria. When this standard applied for esophoric subjects, it met in 25 (83.3\%) and unmet in $5(16.7 \%)$. The mean difference size of esophoria in subjects who met and unmet was not significant $p=0.572$. Concerning (opposing fusional reserve) NFV the mean difference to blur, break and recovery points were statistically significant as shown in Table 6.

Table 6: Sheared and percival criteria among esophoric subjects

\begin{tabular}{|c|c|c|c|}
\hline Sheared criterion & $\begin{array}{l}\text { Successful in } \\
\text { diagnosis } 25(83.3 \%)\end{array}$ & $\begin{array}{l}\text { Failed in diagnosis } \\
5(16.7 \%)\end{array}$ & $p$-value \\
\hline Variable & [Mean \pm SD] & [Mean \pm SD] & \\
\hline Size of esophoria & {$[5.1 \pm 5.8 \Delta$ base-out $]$} & {$[3.6 \pm 2.6 \Delta$ base-out } & 0.572 \\
\hline NFV (blur point) & {$[14.3 \pm 9.1 \Delta$ base-in $]$} & {$[4.4 \pm 2.6 \Delta$ base-in] } & 0.025 \\
\hline NFV (breakpoint) & {$[16.8 \pm 8.7 \Delta$ base-in $]$} & {$[7.2 \pm 3.0 \Delta$ base-in $]$} & 0.022 \\
\hline NFV (recovery point) & {$[11.7 \pm 8.5 \Delta$ base-in] } & {$[2.6 \pm 2.3 \Delta$ base-in $]$} & 0.026 \\
\hline Percival criterion & $\begin{array}{l}\text { Successful in } \\
\text { diagnosis } 26(86.7 \%)\end{array}$ & $\begin{array}{l}\text { Failed in diagnosis } \\
4(13.3 \%)\end{array}$ & $p$-value \\
\hline Variable & {$[$ Mean \pm SD] } & {$[$ Mean \pm SD] } & \\
\hline Size of esophoria & {$[4.5 \pm 4.7 \Delta$ base-out $]$} & [8.0 $\pm 10.4 \Delta$ base-out] & 0.294 \\
\hline NFV (blur point) & {$[12.9 \pm 9.6 \Delta$ base-in] } & {$[11.0 \pm 4.6 \Delta$ base-in $]$} & 0.747 \\
\hline NFV (breakpoint) & {$[15.4 \pm 9.2 \Delta$ base-in $]$} & {$[13.3 \pm 3.1 \Delta$ base-in] } & 0.701 \\
\hline NFV (recovery point) & {$[10.4 \pm 8.9 \Delta$ base-in $]$} & {$[10.2 \pm 8.5 \Delta$ base-in $]$} & 0.749 \\
\hline
\end{tabular}

\section{Percivals criterion among exophoric and} esophoric subjects

Percival postulate that PFV for exophoria should not be less than half NFV to be compensated. When this principle applied for exophoric subjects it met in 147 (73.5\%) and unmet in 53 (26.5\%). Regarding PFV the mean difference to blur, recovery, and breakpoints was statistically significant as shown in Table 5.

Percival assumed that NFV for esophoria should not be less than half PFV to be compensated, when this principle applied for esophoric subjects it met in 26 (86.7\%) and unmet in 4 (13.3\%). Regarding NFV the mean difference to blur, recovery, and breakpoints was not statistically significant as shown in Table 6.

\section{Sensitivity and specificity of the sheard criteria in exophoric and esophoric subjects}

Sheard's criteria showed a high degree of agreement in the diagnosis of exophoria, with a sensitivity of $87.2 \%$ and a specificity of $8.0 \%$ with a positive and negative predictive value of $65.5 \%$ and $26 \%$, respectively. While the criteria showed a relatively low level of agreement in the diagnosis of esophoria with a sensitivity of $77.8 \%$ and a specificity of $9.0 \%$ with a positive and negative predictive value of $56 \%$ and $20 \%$, respectively, Table 2 .

\section{Sensitivity and specificity of the percival criterion in exophoric and esophoric individuals}

The percival criteria showed a high degree of agreement in the diagnosis of esophoria with a sensitivity of $84.2 \%$ and a specificity of $9.1 \%$ with a positive and negative predictive value of $61.5 \%$ and $25 \%$, respectively. While the criteria showed low degree of agreement in diagnosis of exophoria with sensitivity of $67.4 \%$ and specificity of $13.8 \%$ with positive and negative predictive value of $61.9 \%$ and $17 \%$, respectively, Table 3 . 


\section{Discussion}

This study demonstrates that Sheard's criterion with high sensitivity and low specificity in diagnosis exophoria. The criterion showed a relatively low level of sensitivity and specificity in diagnosis esophoria. However, Percival's criterion revealed high sensitivity and low specificity in diagnosis esophoria whereas the criterion showed low sensitivity and specificity in diagnosis exophoria.

When heterotopy is present, the magnitude of the deviation exceeds the capabilities of the fusional vergence amplitude, the heterophoria is probably decompensated. There are physiological states of the visual system that lend themselves to direct clinical measurement. Consequently, the current study was required to determine the point at which the relevant fusional vergence reserve is insufficient to support heterophoria, resulting in decompensated phoria or patients becoming symptomatic.

Sheard assumed that symptoms of heterophoria could be avoided if the fusion vergence in the opposite direction was at least twice that of phoria $(11,26)$. In this study, Sheard's criteria showed a high sensitivity of $87.2 \%$ and a low specificity of $8.0 \%$ for the diagnosis of exophoria. The mean magnitude of exophoria in subjects who met and did not meet the criteria was [5.2 $\pm 4.8 \Delta$ base-in] and [5.7 $\pm 2.2 \Delta$ base-in], respectively, which was not significant $p=0.623$. Regarding the contrast between fusion vergence and exophoria, the mean difference between PFV to blur was not significant $p=0.608$ in subjects who met and did not meet Sheard's criterion. However, PFV to breakpoint was significant $p$ $=0.021$ in exophoric subjects who did and did not meet Sheard's criterion. Nevertheless, the mean difference between those who met and did not meet for PFV to recovery point was statically significant $p=0.021$ Table 5. This result indicated that the clinical value for a breakpoint is more accurate than the bur and recovery point in assessing fusion vergence for the subjects with exophoria. Conversely, Pickwell [1] reported that the recovery point should be within $4-6 \Delta$ of the breakpoint, a recovery point worse than this could be a sign of decompensated heterophoria. Yu et al., [27] reported that the diagnosis of exophoria according to Sheard's criteria is the best way to evaluate convergence insufficiency. The concept of fusional reserve implies that PFV represents resources to overcome exophoria. Several studies [26], [27], [28], [29] have shown that symptoms of exophoria are significantly reduced or eliminated by increasing PFV and/or reducing exophoria.

Sheard's criterion showed relatively low agreement in diagnosing esophoria in this study, with a sensitivity of $77.8 \%$ and a low specificity of $9.0 \%$. The mean difference of the magnitude of esophoria in subjects meeting and not meeting the Sheard criterion was not significant $p=0.572$. Regarding the opposite fusion vergence NFV, the mean difference to blur, break and recovery points was $p=0.025, p=0.022$, and $p=0.026$, respectively, which was statistically significant. Myklebust and Riddell reported that there is a risk of missing decompensated heterophoria based only on passing Sheard's criterion and recommended that a continuous fusion persistence alternative may be useful for clinicians in quantifying binocular vision problems and monitoring treatment effects [12]. However, Moon et al. [8] stated that Sheard's criterion is a useful tool for screening convergence insufficiency with exophoria associated with near vision activities. Therefore, Sheard's criterion is not sensitive enough to detect the binocular problems alone without another diagnostic test [30]. Accordingly, Sheard's criterion can provide good guidance and predictions for the diagnosis of individuals with decompensated heterophoria.

In the current study, the Percival criterion showed a high sensitivity of $84.2 \%$ and a low specificity of $9.1 \%$ for the diagnosis of esophoric individuals. In terms of opposite fusion vergence NFV, the mean difference on blur, breakage and recovery in esophoric patients who met and did not meet the Percival criterion was $p=0.747, p=0.701$ and $p=0.749$, respectively, which was not significant Table 5 . This indicates that the Percival criterion is a very sensitive tool for diagnosing esophoria. This is in line with the previous studies [1], [9] which indicated that the Percival criterion is a good predictor of binocular problems in esophoric subjects. However, the criterion showed a low sensitivity of $67.4 \%$ and specificity of $13.8 \%$ in diagnosing exophoria. In terms of opposite fusion vergence PFV, the mean difference between fusion reserves for blur, break and recovery in exophoric patients who met and did not meet the Percival criteria was $p=0.001, p=0.002$, and $p=0.001$, respectively, which was significant Table 5. This suggests that the Percival criteria are not a sensitive means of diagnosing exophoria. This study has some limitations. The sample size for esophoria was small, for future studies it is recommended to increase the sample size for esophoric subjects; to apply the Sheard and Percival criteria for the diagnosis of convergence weakness exophoria and to evaluate the sensitivity and specificity of the Sheard and Percival criteria to determine the amount of prism or lens to alleviate symptoms for binocular vision problems.

\section{Conclusion}

The study shows that Sheard's and Percival's criteria are useful tools for diagnosing binocular vision problems. Sheard's criteria are more accurate in diagnosing near exophoria and Percival's criteria are more precise in diagnosing near esophoria. In 
addition, this study showed that PFV to blur showed no significant change in exophoric patients who did and did not meet Sheard's criteria. However, PFV to the breakpoint showed a significant change in exophoric patients who did and did not meet Sheard's criteria. While NFV to blur and break showed no significant change in esophoric patients who met and did not meet Percival's criteria. Therefore, the Sheard and Percival criteria provide good guidance and predictions for the diagnosis of binocular vision problems.

\section{References}

1. Evans BW. Pickwell's Binocular Vision Anomalies: Investigation and treatment. $5^{\text {th }}$ ed. London: Butterworth-Heinemann; 2007. p. 58-91. https://doi.org/10.1016/b978-0-7506-8897-0.50007-X

2. Ansons $A M$, Davis $H$. Diagnosis and Management of Ocular Motility Disorders. $1^{\text {th }}$ ed. Oxford: Wiley Blackwell; 2014. p. $30-75$

3. Scheiman M, Wick B. Clinical Management of Binocular Vision: Heterophoric, Accommodative, and Eye Movement Disorders. Philadelphia, PA: Lippincott Williams and Wilkins; 2008. p. 75-90. https://doi.org/10.1111/opo.12161

4. Rosenfield M, Logan N. Optometry: Science, Techniques and Clinical Management. Amsterdam, Netherlands: Elsevier Health Sciences; 2009. p. 28-60.

5. London R, Crelier RS. Fixation disparity analysis: Sensory and motor approaches. Optometry. 2006;77(12):590-608. https:// doi.org/10.1016/j.optm.2006.09.006

PMid:17157241

6. Kim SY, Cho HG, Moon BY, Yu DS. Fusional single vision with prism-induced vergence has more influence than diplopia on postural stability. Optom Vis Sci. 2020;97(3):218-26. https://doi. org/10.1097/opx.0000000000001483

PMid:32168245

7. Benjamin WJ. Borish's Clinical Refraction-E-Book. Amsterdam, Netherlands: Elsevier Health Sciences; 2006. p. 30-50.

8. Moon BY, Kim SY, Yu DS. Receiver operating characteristic curve analysis of clinical signs for screening of convergence insufficiency in young adults. PLoS One. 2020;15(1):e0228313. https://doi.org/10.1371/journal.pone.0228313 PMid:31978203

9. Evans BJ. Optometric prescribing for decompensated heterophoria. Optom Pract. 2008;9(2):63-78.

10. Alrasheed SH, Naidoo KS, Clarke-Farr PC. Prevalence of visua impairment and refractive error in school-aged children in South Darfur State of Sudan. Afr Vis Eye Health. 2016;75(1):1-9. https://doi.org/10.4102/aveh.v75i1.355

11. Lança CC, Rowe FJ. Measurement of fusional vergence: A systematic review. Strabismus. 2019;27(2):88-113. https://doi. org/10.1080/09273972.2019.1583675 PMid:30821611

12. Myklebust A, Riddell P. Fusional stamina: An alternative to Sheard's criterion. Scand J Optom Vis Sci. 2016;9(2):1-7. https://doi.org/10.5384/sjovs.vol9i2p1-7

13. Scheiman M, Cotter S, Rouse M, Mitchell GL, Kulp M, Cooper J, et al. Randomized clinical trial of the effectiveness of base-in prism reading glasses versus placebo reading glasses for symptomatic convergence insufficiency in children. $\mathrm{Br} J$
Ophthalmol. 2005;89(10):1318-23. https://doi.org/10.1136/ bjo.2005.068197

PMid:16170124

14. Casillas EC, Rosenfield M. Comparison of subjective heterophoria testing with a phoropter and trial frame. Optom Vis Sci. 2006;83(4):237-41. https://doi.org/10.1097/01. opx.0000214316.50270.24

PMid:16614580

15. Rainey BB, Schroeder TL, Goss DA, Grosvenor TP Inter-examiner repeatability of heterophoria tests. Optom Vis Sci. 1998;75(10):719-26. https://doi. org/10.1097/00006324-199810000-00016 PMid:9798211

16. Scheiman M, Talasan $H$, Alvarez TL. Objective assessment of disparity vergence after treatment of symptomatic convergence insufficiency in children. Optom Vis Sci. 2019;96(1):3-16. https:// doi.org/10.1097/opx.0000000000001320

PMid:30570596

17. Alvarez TL, Scheiman M, Santos EM, et al. The convergence insufficiency neuro-mechanism in adult population study (CINAPS) randomized clinical trial: Design, methods, and clinical data. Ophthalmic Epidemiol. 2020;27(1):52-72. https:// doi.org/10.1080/09286586.2019.1679192

PMid:31640452

18. Alrasheed SH. Effect of binocular vision problems on childhood academic performance and teachers' perspective. Pak J Ophthalmol. 2020;36(2):163-8. https://doi.org/10.36351/pjo. v36i2.896

19. Sheedy JE, Saladin JJ. Phoria, vergence, and fixation disparity in oculomotorproblems.Am J OptomPhysiolOpt. 1977;54(7):474-8 https://doi.org/10.1097/00006324-197707000-00008 PMid:931014

20. Darko-Takyi C, Moodley VR, Boadi-Kusi SB. A review of normative data for parameters of functional non-strabismic binocular vision. Afr Vis Eye Health. 2020;79(1):1-4. https://doi. org/10.4102/aveh.v79i1.507

21. Alrasheed $\mathrm{SH}$. Clinical characteristics of patients with headache attending the binocular vision clinic at Al-Neelain eye hospital. Pak J Ophthalmol. 2020;36(3):247-52. https://doi.org/10.36351/ pjo.v36i3.1046

22. Fray KJ. Fusional amplitudes: exploring where fusion falters. Am Orthopt J. 2013;63(1):41-54. https://doi.org/10.3368/aoj.63.1.41 PMid:24141750

23. Lança CC, Rowe FJ. Variability of fusion vergence measurements in heterophoria. Strabismus. 2016;24(2):63-9. https://doi.org/10. 3109/09273972.2016.1159234

PMid:27128321

24. Stojanović $M$, Apostolović $M$, Stojanović $D$, Milošević $Z$, Toplaović A, Mitić-Lakušić V, et al. Understanding sensitivity, specificity and predictive values. Vojnosanit Pregl. 2014;71(11):1062-5. https://doi.org/10.2298/vsp1411062s PMid:25536811

25. Ying GS, Maguire MG, Glynn RJ, Rosner B. Calculating sensitivity, specificity, and predictive values for correlated eye data. Investig Ophthalmol Vis Sci. 2020;61(11):29-33. https:// doi.org/10.1167/iovs.61.11.29

PMid:32936302

26. Rowe FJ. Fusional vergence measures and their significance in clinical assessment. Strabismus. 2010;18(2):48-57. https://doi. org/10.3109/09273971003758412

PMid:20521879

27. Yu DS, Cho HG, Moon BY. Influence of different diagnostic criteria on frequency of convergence insufficiency. J Korean Ophthalmic Opt Soc. 2016;21(3):235-42. 
28. Atzmon D, Nemet $P$, Ishay A, Karni E. A randomized prospective masked and matched comparative study of orthoptic treatment versus conventional reading tutoring treatment for reading disabilities in 62 children. Bin Vis Eye Muscle Surg Q. 1993;8(2):91-106.

29. Aziz S, Cleary M, Stewart HK, Weir CR. Are orthoptic exercises an effective treatment for convergence and fusion deficiencies? Strabismus. 2006;14(4):183-9. https://doi. org/10.1080/09273970601026185

PMid:17162439

30. Davis AL, Harvey EM, Twelker JD, Miller JM, Leonard-Green T, Campus I. Convergence insufficiency, accommodative insufficiency, visual symptoms, and astigmatism in Tohono O'odham students. J Ophthalmol. 2016;2016:6963976. https:// doi.org/10.1155/2016/6963976

PMid:27525112 